\title{
PROJETO E IMPLEMENTAÇÃO DE UM REATOR ELETRÔNICO MICROCONTROLADO, DE ELEVADO FATOR DE POTÊNCIA, ORIENTADO A LÂMPADAS DE VAPOR METÁLICO
}

\author{
Marco Antônio Dalla Costa* \\ madc.smegmail.com
}

Henrique A. C. Braga
henrique.bragaeufjf.edu.br

\author{
Marina M. S. D. Perdigão ${ }^{\dagger}$ \\ perdigaodisec.pt \\ José Marcos Alonso ${ }^{\S}$ \\ marcos@uniovi.es
}

\author{
*Universidade de Caxias do Sul - Caxias do Sul, Brasil \\ ${ }^{\dagger}$ Instituto de Telecomunicações - Coimbra, Portugal
}

${ }^{\ddagger}$ Núcleo de Iluminação Moderna (NIMO), Universidade Federal de Juiz de Fora, Brasil

$\S$ Area de Tecnología Electrónica, Universidad de Oviedo, Espanha

\section{RESUMO}

Este trabalho descreve a análise, o projeto e a implementação de um reator eletrônico para lâmpadas de vapor metálico $(\mathrm{MH})$ baseado em microcontrolador. O circuito do reator se fundamenta na integração de um conversor buck com um conversor flyback. A tarefa de correção do fator de potência é realizada pela etapa buck, enquanto a etapa flyback controla a potência na lâmpada fornecendo uma corrente em onda quadrada de baixa frequiência. Este é um método adequado para se evitar o fenômeno da ressonância acústica, comum em lâmpadas de descarga de alta pressão (HID). Os dois estágios conversores operam no modo de condução descontínua (DCM), o que permite o uso de apenas um interruptor de alta frequiência simplificando o controle. O reator eletrônico é controlado digitalmente e emprega um microcontrolador de baixo custo PIC16F684. Este componente é programado para desempenhar todas as tarefas necessárias aos processos de ignição, aquecimento e regime permanente da lâmpada, respondendo também pelo controle em malha fechada da corrente na lâmpada e proteções convencionais. $\mathrm{O}$ artigo inclui resultados experimentais obtidos de um pro-

Artigo submetido em 01/12/2008 (Id.: 00928)

Revisado em 10/02/2009, 01/04/2009

Aceito sob recomendação do Editor Associado Prof. Enes Gonçalves Marra tótipo que alimenta uma lâmpada MH de 35W.

PALAVRAS-CHAVE: Controle de iluminação, microcontroladores, lâmpada de vapores metálicos.

\section{ABSTRACT}

The analysis, design and implementation of a microcontroller-based electronic ballast to supply metal halide $(\mathrm{MH})$ lamps are presented. The proposed power scheme is based on the integration of the buck and flyback converters. The former providing power factor correction and the latter controlling lamp power by supplying the lamp with a low frequency square waveform current, which is a convenient way to avoid acoustic resonances in high intensity discharge (HID) lamps. Both converters operate in discontinuous conduction mode (DCM), thus allowing the use of only one high-frequency switch and simplifying the control. The electronic ballast is digitally controlled by using a low cost microcontroller PIC16F684. The microcontroller performs all the necessary tasks during starting, warming-up and steady state, including closed loop control of lamp current and protections. Experimental results for a 35W MH lamp are presented. 
KEYWORDS: Lighting control, microcontrollers, metal halide lamps.

\section{INTRODUÇÃO}

Os reatores eletrônicos para lâmpadas de descarga de alta pressão (HID) atraíram a atenção por causa de suas vantagens, comparadas aos reatores eletromagnéticos, como: menor peso, tamanho reduzido, eficiência mais elevada, e melhor imunidade às mudanças da tensão da rede. O inconveniente principal de usar reatores eletrônicos orientados a lâmpadas HID consiste na possibilidade de se provocar ressonâncias acústicas (AR) (Yan et al., 2001). A ressonância acústica é um fenômeno que produz a geração de ondas instáveis de pressão no plasma, tendo por resultado a flutuação do arco no interior do bulbo que, por sua vez, pode causar cintilação luminosa ou mesmo a extinção do arco (de Groot \& van Vliet, 1986; Dalla Costa et al., 2007a). Trabalhos científicos recentes indicam que a melhor solução para evitar o problema de AR é fornecer à lâmpada uma corrente de onda quadrada de baixa freqüência (LFSW), porque a potência instantânea teórica desta forma de onda é constante, o que evita a AR (Dalla Costa et al., 2007b; Shen et al., 2003).

Este artigo é focalizado nas lâmpadas MH de baixa potência (Marques et al., 2007), que são particularmente sensíveis ao efeito da AR. Por este motivo, será considerado o uso de um reator eletrônico LFSW.

Reatores LFSW convencionais, entretanto, apresentam uma relativa complexidade, pois empregam três etapas conversoras, que executam as tarefas de: 1) correção do fator de potência (PFC), 2) controle de potência, e 3) conversão CCCA (inversão); resultando em uma alternativa dispendiosa. Por isso, muita pesquisa tem sido desenvolvida no sentido de integrar estes estágios (Shen et al., 2003; Có et al., 2003; Dalla Costa et al., 2005; Li et al., 2004; Yang et al., 2005; Wu et al., 2005; Zobaa, 2006; Bhat \& Venkatraman, 2005; Marchesan et al., 2007), o que geralmente resulta em circuitos complexos apresentando esforços significativos de tensão ou corrente nos interruptores compartilhados. Além disso, o comportamento da lâmpada MH também aumenta a complexidade do circuito de controle, uma vez que estas lâmpadas têm diferentes fases operacionais, que podem ser classificadas em:

Partida da Lâmpada - a lâmpada apresenta elevada impedância antes da ignição, e um pulso de aproximadamente 3 $\mathrm{kV}$ é necessário para promover a ignição de uma lâmpada fria (quando quente, seria necessário um pulso de cerca de $30 \mathrm{kV})$;

Aquecimento da Lâmpada - o processo de aquecimento leva desde dezenas de segundos até minutos, quando a lâmpada apresenta uma pequena impedância inicial, que aumenta enquanto a lâmpada é aquecida. Esta fase deve ser a mais curta possível, a fim de evitar o efeito prejudicial da corrente de partida (glow current);

Regime Permanente - após o aquecimento da lâmpada, atinge-se o estado estacionário, e algum parâmetro (e.g. potência da lâmpada ou corrente) deve ser controlado.

Sabe-se que muitos reatores convencionais, em especial os eletromagnéticos, não promovem qualquer ajuste de desempenho no sentido de compensar as variações do comportamento da lâmpada, preservar a sua vida útil e garantir a qualidade de iluminação enquanto a unidade estiver sendo empregada. Entretanto, não é difícil concluir que a incorporação destas funcionalidades torna-se fundamental quando se deseja maximizar o investimento realizado ou garantir um nível de iluminamento mais homogêneo ao longo da vida útil do produto.

Devido à complexidade do circuito de controle destinado a implantar tais funções, o emprego de um sistema baseado em microcontrolador é recomendado, já que confere diversas vantagens se comparado aos métodos analógicos tradicionais. Microcontroladores têm sido usados em propósitos similares, conforme reporta a literatura técnica recente (Lee \& Cho, 2003). Além disso, um sistema controlado digitalmente apresenta confiabilidade elevada e maior flexibilidade. Imunidade a ruído, tolerância a fatores ambientais, possibilidade de mudar o esquema do controle sem modificar o circuito e o custo baixo são outras características atrativas dos controladores digitais (Kutluay et al., 2006; Buso et al., 1998; Feng et al., 2006).

Neste artigo, a seção II descreve a topologia do reator proposto, ao passo que a seção III define os estágios de operação do reator e as exigências do circuito de controle. Na sequiência, a seção IV apresenta a descrição do circuito de controle e a seção V mostra os resultados experimentais. Por fim, as conclusões do trabalho são apresentadas na seção VI.

\section{O REATOR PROPOSTO}

A Figura 1 mostra a topologia do reator empregada neste trabalho, que foi apresentado originalmente em Dalla Costa et al. (2005). Este circuito é obtido pela integração de um conversor (ou pré-regulador) buck, que controla o fator de potência, com um conversor flyback que, por sua vez, controla a potência na lâmpada, evitando esforços adicionais de corrente no interruptor principal. O indutor do conversor flyback inclui dois enrolamentos secundários que comutam complementarmente em baixa freqüência, a fim de fornecer à lâmpada uma corrente LFSW. Ambos os conversores operam no modo de condução descontínua (DCM) e compartilham o 


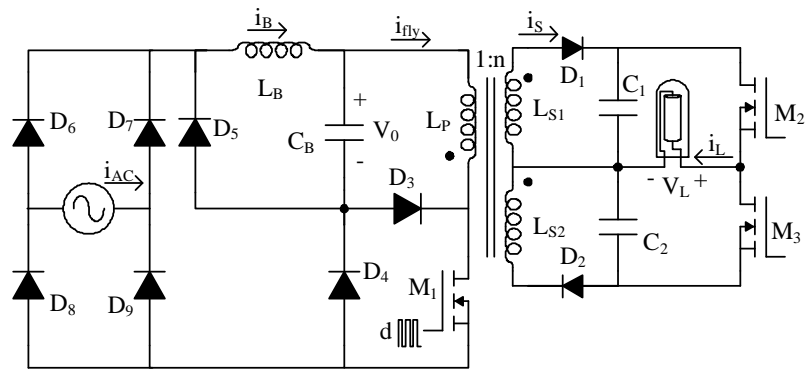

Figura 1: Reator proposto.

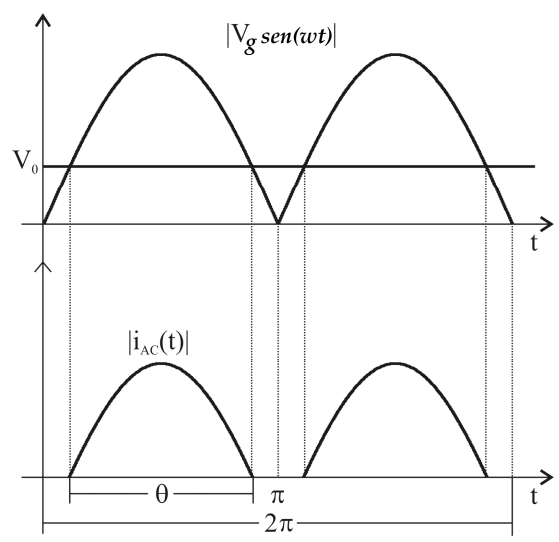

Figura 2: Formas de onda teóricas do conversor buck. (a) Tensão de linha retificada e tensão em CB; (b) Corrente na saída do retificador.

mesmo interruptor $\left(M_{1}\right)$. Os capacitores $C_{1}$ e $C_{2}$ são empregados para filtrar a componente alternada da corrente na lâmpada. As características adicionais deste circuito são detalhadas em Dalla Costa et al. (2005). O princípio da aplicação do conversor buck na melhoria do fator de potência de pré-reguladores encontra-se bem detalhado em Alonso et al., 2004 e pode ser ilustrado pelas formas de onda apresentadas na Figura 2, que mostram a tensão de linha retificada (|Vg sen $(\omega t) \mid)$, a tensão da saída do conversor buck $\left(V_{o}\right)$, e a corrente de linha filtrada $\left(\left|i_{A C}(t)\right|\right)$.

Como pode ser visto, o conversor buck comporta-se como uma carga resistiva do ponto de vista da rede quando a tensão de linha instantânea é mais elevada do que a tensão da saída do conversor buck, $V_{o}$. Conseqüentemente, o valor de $V_{o}$ deve ser projetado a fim de limitar as componentes harmônicas de $i_{A C}$ de acordo com os limites impostos pela norma IEC 61000-3-2 (classe C). Para tal, deve-se garantir um ângulo mínimo de condução de $130^{\circ}$ (i. e., $\theta>130^{\circ}$ ), de acordo com Alonso et al., 2004. Assim, os componentes do conversor buck, $L_{B}$ e $C_{B}$, devem ser calculados a fim de impor $V_{o}$ $<137 \mathrm{~V}$, o que significa $\theta>130^{\circ}$.

Isto pode ser alcançado a partir da expressão (1), que permite definir a resistência equivalente do conversor buck $\left(R_{S}\right)$ para uma tensão de saída $\left(V_{O}\right)$.

$$
\begin{aligned}
\frac{V_{g}^{2}}{2 R_{S} V_{o}}\left[1-\frac{2}{\pi} \operatorname{sen}^{-1}\left(\frac{V_{o}}{V_{g}}\right)\right]- & \\
& -\frac{1}{\pi R_{S}} \sqrt{V_{g}^{2}-V_{o}^{2}}-\frac{V_{o}}{R_{f l y}}=0 .
\end{aligned}
$$

em que $V_{g}$ é a tensão de pico da rede, $R_{S}$ a resistência

equivalente de saída do conversor, e $R_{f l y}$ a resistência equivalente de carga do conversor flyback, que são definidas em (2) e (3), respectivamente.

$$
\begin{gathered}
R_{S}=\frac{2 L_{B} f_{s}}{d^{2}} . \\
R_{f l y}=\frac{2 L_{P} f_{s}}{d^{2}} .
\end{gathered}
$$

em que $L_{B}$ é a indutância buck, $L_{P}$ a indutância do enrolamento primário do conversor flyback, $f_{S}$ é a freqüência de comutação, e $d$ a razão cíclica.

O comportamento do conversor flyback, que trabalha no modo descontínuo e é alimentado por uma tensão $\mathrm{CC}, V_{o}$, também é bem conhecido na literatura, e seu projeto completo pode ser encontrado em Alonso et al. (1997). Este conversor trabalha como uma fonte de corrente, e um único inversor de meia-ponte $\left(M_{2}\right.$ e $\left.M_{3}\right)$ é responsável por comutar, em baixa frequiência $\left(f_{B F}=100\right.$ hertz), os enrolamentos secundários do conversor. Os capacitores $C_{1}$ e $C_{2}$, de baixa capacitância, são empregados para filtrar a ondulação de corrente que é imposta pela operação DCM do conversor flyback.

O valor crítico da razão cíclica, $d_{c r i t}$, que define o limite entre a operação DCM e a operação contínua (CCM), é definido em (4). A corrente média da lâmpada, $I_{L}$, é expressa por (5). Os componentes do reator foram projetados de acordo com Dalla Costa et al., 2005, para $V_{o}=100 \mathrm{~V}$, e seus valores são apresentados na Tabela I.

$$
\begin{gathered}
d_{\text {crit }}=\frac{1}{1+\frac{n \cdot V_{o}}{V_{L}}} . \\
I_{S_{-} \text {avg }}=\frac{V_{o}^{2} d^{2} T_{S}}{2 V_{L} L_{P}} .
\end{gathered}
$$


Em que $T_{S}$ é o período de chaveamento, $n$ é a relação de espiras do conversor flyback $\left(n=\sqrt{\mathrm{L}_{\mathrm{S}} / \mathrm{L}_{\mathrm{P}}}\right)$, e $V_{L}$ é a tensão eficaz da lâmpada.

\section{EXIGÊNCIAS NA OPERAÇÃO DA LÂM- PADA}

O comportamento do reator proposto, considerando a lâmpada como uma resistência ideal, é bastante simples e requer uma metodologia de controle muito trivial. Entretanto, as lâmpadas MH têm exigências de operação especiais durante a partida, aquecimento e mesmo em regime, que devem ser levadas em consideração no projeto do sistema de controle.

Tabela 1: Parâmetros do Reator Eletrônico.

\begin{tabular}{|c|c|}
\hline Parâmetro & Valor \\
\hline Rede & 230Vrms, $50 \mathrm{~Hz}$ \\
\hline$D_{1}-D_{9}$ & MUR160 \\
\hline$M_{1}-M_{3}$ & BUK456 \\
\hline$L_{B}$ & $434 \mu \mathrm{H}$ (núcleo EF25) \\
\hline$C_{B}$ & $220 \mu \mathrm{F} / 200 \mathrm{~V}$ \\
\hline$L_{P}, L_{S 1}, L_{S 2}$ & $180,315,315 \mu \mathrm{H}$ (núcleo EF25) \\
\hline$C_{1}, C_{2}$ & $220 \mathrm{nF} / 500 \mathrm{~V}$ \\
\hline Lâmpada & Philips CDM-T35W \\
\hline$V_{L}, I_{L}, R_{L}$ & $90 \mathrm{~V} ; 0,42 \mathrm{~A} ; 215 \Omega$ \\
\hline$f_{S}, T_{S}$ & $85 \mathrm{kHz}, 11.75 \mu \mathrm{s}$ \\
\hline$f_{B F}$ & $100 \mathrm{~Hz}$ \\
\hline
\end{tabular}

\subsection{Partida da Lâmpada}

A partida da lâmpada consiste em três fases: colapso dos gases, ionização inicial e estabelecimento do arco. Uma alta tensão de ignição é necessária para fornecer o colapso elétrico dos gases no interior do bulbo. Esta tensão é da ordem de $3 \mathrm{kV}$ com lâmpada fria. Geralmente, o colapso dos gases faz com que uma descarga inicial ocorra, sendo que a duração deve ser minimizada a fim aumentar a vida útil da lâmpada. O fim do processo de partida ocorre com a transição da ionização inicial para o estabelecimento definitivo do arco, quando a alta tensão e os baixos valores de corrente dão lugar a uma baixa tensão e valores de correntes mais altos (descarga termiônica), devido à ionização contínua.

\subsection{Aquecimento}

Nesta fase, as contínuas colisões de elétrons mantêm a temperatura do arco em elevação, o que vaporiza os elementos metálicos e aumentam a pressão no interior do bulbo. Du-

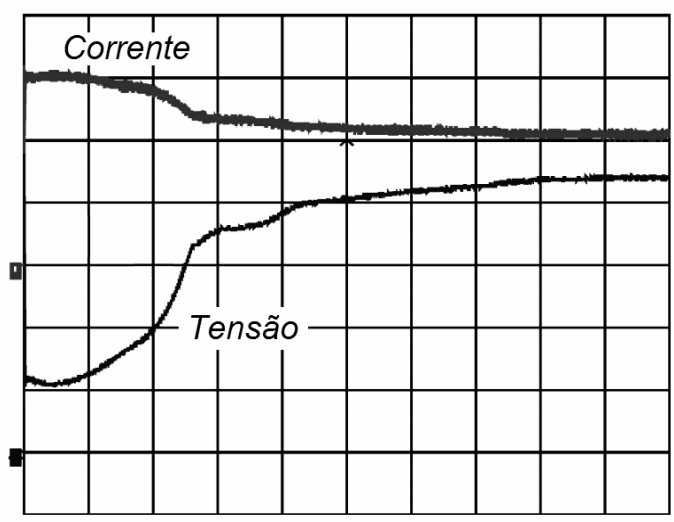

Figura 3: Processo de aquecimento da lâmpada (20s/div). Em cima: corrente eficaz ( $0,2 \mathrm{~A} / \mathrm{div})$; em baixo: tensão eficaz terminal (20V/div)

rante este estágio, que pode durar desde dezenas de segundos até alguns minutos, a tensão da lâmpada aumenta e a corrente da lâmpada deve ser limitada a um valor apropriado. A Figura 3 mostra a evolução típica da tensão e da corrente na lâmpada nesta fase. Finalmente, quando o arco definitivo de alta pressão é estabelecido, a temperatura e o brilho na lâmpada alcançam seus valores nominais.

Durante a vida útil da lâmpada, sua tensão terminal aumenta e ocorre uma redução do nível de iluminamento. Estes fatores devem ser levados em conta a fim de se definir a estratégia de controle. Além disso, o comportamento dinâmico da lâmpada deve ser considerado para projetar o controle em malha fechada. Um modelo dinâmico satisfatório da lâmpada é dado por uma função de transferência com um pólo e um zero de acordo com Deng \& Æuk (1997):

$$
z_{L}(s)=\frac{v_{L}(s)}{i_{L}(s)}=k \frac{s+z}{s+p},
$$

em que $v_{L(s)}$ e $i_{L(s)}$ são as transformadas de Laplace da tensão e corrente na lâmpada, respectivamente. Este modelo será usado em associação ao modelo de pequenos sinais do conversor a fim de se programar um controlador digital adequado, por meio do software interno do microcontrolador.

\section{A ESTRATÉGIA DE CONTROLE PRO- POSTA}

Esta seção descreve a estratégia de controle proposta que deverá ser executada pelo microcontrolador. O microcontrolador PIC16F684, de baixo custo, é suficiente para suprir as exigências impostas pela operação da lâmpada. A Figura 4 mostra o circuito esquemático do reator eletrônico proposto 
incluindo os sensores e os circuitos integrados dedicados ao controle e disparo do inversor em meia-ponte. $\mathrm{O}$ circuito de ignição é baseado em um dispositivo centelhador (SG) que aplica os pulsos de ignição quando a lâmpada está desligada. O capacitor $C_{i g n}$ é carregado através de $R_{i g n}$ até que a

tensão de limiar do centelhador $(250$ - 300V) seja atingida. Em seguida, esta tensão é aplicada nos terminais do enrolamento primário do transformador e elevada de acordo com a relação de transformação $n_{i g n}=15$. O monitoramento dos principais

parâmetros do circuito é realizado por meio dos sensores resistivos: a tensão de barramento, $V_{B U S}$, através de um divisor de tensão dado por $R_{B 1}$ e por $R_{B 2}$; a corrente da lâmpada, $i_{L}$, por uma resistência $R_{i l}$ em série com $M_{3}$; e a corrente principal do interruptor pela resistência série $R_{\text {ilim }}$. Conseqüentemente, como todas as medidas são referenciadas ao terra do circuito, nenhum dispositivo de isolamento é necessário. Os interruptores $M_{2}$ e $M_{3}$ são acionados por meio de um circuito integrado convencional, IR2111, controlado por uma porta de saída do microcontrolador. As subseções seguintes descrevem sistematicamente a estratégia de controle.

\subsection{Partida da Lâmpada}

Antes da partida da lâmpada o conversor flyback opera como se estivesse sem carga. Neste caso, a tensão de saída deste conversor, $V_{B U S}\left(V_{B U S}=V_{C 1}+V_{C 2}\right)$ começa a crescer.

Nesta etapa, o microcontrolador controla a razão cíclica de M1 $(d)$ a fim de limitar $V_{B U S}$ abaixo da tensão de ruptura dos interruptores $M_{2}$ e $M_{3}$ (que é de $800 \mathrm{~V}$ ). Então, o circuito centelhador aplica o pulso de ignição nos terminais da lâmpada e, se a lâmpada parte em definitivo, $V_{B U S}$ cai rapidamente. Quando o microcontrolador detecta esta queda da tensão, ele determina um aumento da razão cíclica, a fim manter a descarga e aquecer a lâmpada tão rapidamente quanto possível. Se o processo de ignição falhar e $V_{B U S}$ aumentar outra vez, o microcontrolador retorna $d$ ao valor mínimo, e um contador de falha (F) é incrementado.

Definiu-se que quando este contador de falha alcança um valor igual a 10, o conversor deve esperar 5 minutos antes de reiniciar o processo, a fim de permitir um resfriamento natural da lâmpada (o reator descrito neste trabalho não foi projetado para propiciar a ignição de uma lâmpada quente). Caso se estabeleça esta condição de espera, um outro contador $(\mathrm{G})$ é incrementado para gravar o evento. Se $\mathrm{G}$ alcançar um valor igual a 5, o processo de partida é interrompido e um LED indicador é aceso para avisar sobre a necessidade de substituição da lâmpada. Estes valores foram definidos empiricamente, pela observação da operação típica de reatores pro-

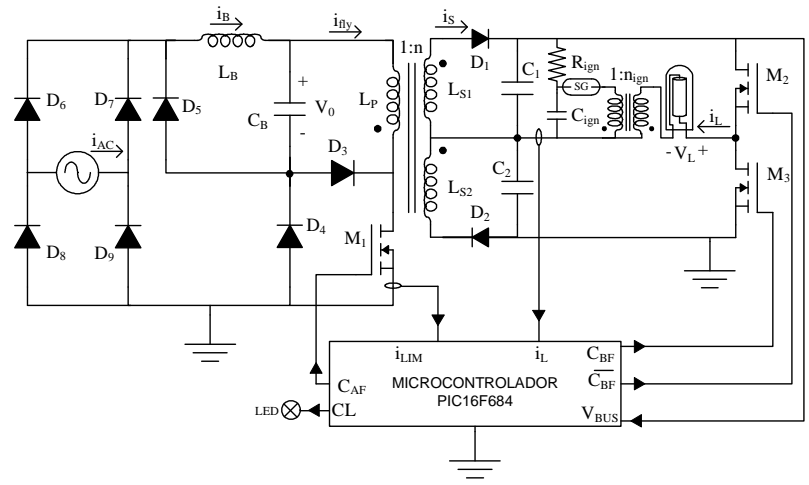

Figura 4: Esquemático do reator implementado, incluindo sensores e CIs dedicados.

jetados para acionar lâmpadas HID, já que não há nenhuma norma aplicável atualmente sobre isso. Não obstante, já que todo o controle é feito usando um microcontrolador, seria muito fácil reajustar estes valores a fim de se atender a outras exigências.

\subsection{Processo de Aquecimento}

Uma vez que a lâmpada passou pela transição da ionização inicial ao arco, sua tensão atinge o valor mínimo (em torno de 20V). Neste estado, o microcontrolador deve estabelecer a razão cíclica $d$ a fim manter a operação DCM do conversor flyback, como determinado em (4). Esta fase termina quando a tensão da lâmpada alcança seu valor nominal (acima de 80 V). Além disso, se a lâmpada desligasse durante esta fase, o microcontrolador esperaria 5 minutos para reiniciar o processo e incrementaria o contador G. A operação durante o processo de partida e de aquecimento é muito instável, o que resulta no desligamento da lâmpada muito facilmente. Conseqüentemente, a fim de melhorar a estabilidade nestas fases, a comutação em baixa freqüência dos interruptores $M_{2}$ e $M_{3}$ não é habilitada, sendo que somente um deles está em condução. Ou seja, nas etapas de partida e aquecimento, a lâmpada é acionada em corrente contínua. A cada novo processo de partida, o interruptor ligado alternará entre $M_{2}$ e $M_{3}$, a fim de garantir o desgaste homogêneo dos elétrodos da lâmpada. O microcontrolador escolhe o interruptor a ser ligado após verificar o contador $\mathrm{H}$, que é armazenado em sua memória não-volátil.

\subsection{Operação em Regime}

A comutação em baixa freqüência de $M_{2}$ e $M_{3}$ é ativada nesta fase. A estratégia proposta permite controlar a corrente da lâmpada $\left(i_{L}\right)$ e/ou a potência na lâmpada, uma vez que a tensão da lâmpada é medida indiretamente através de $V_{B U S}$. Foi selecionado um algoritmo de controle de corrente 
porque, neste caso, o fluxo luminoso da lâmpada pode ser mantido mais constante durante sua vida útil (Dalla Costa $e t$ al., 2006). O sistema lâmpada-reator foi modelado dinamicamente de acordo com Alonso et al. (2007), considerando o efeito da indutância magnetizante do transformador do dispositivo de ignição, o que resulta na seguinte função de transferência:

$$
\begin{aligned}
& G(s)=\frac{i_{L}(s)}{d(s)}=\frac{A}{B} \quad \text { sendo que } \\
& \begin{array}{l}
A=2,454 s+26240 \mathrm{e} \\
B=9,9 \cdot 10^{-9} s^{3}+1,387 \cdot 10^{-4} s^{2}+1,612 s+9654
\end{array}
\end{aligned}
$$

que relaciona a corrente da lâmpada com a razão cíclica do interruptor $M_{1}$. O diagrama de blocos do sistema de controle é mostrado na Figura 5, onde $G_{R}(z)$ é a função de transferência do controlador digital, $G(s)$ representa a função de transferência do reator e lâmpada, e $H(s)$ corresponde ao ganho de realimentação do sensor de corrente da lâmpada.

O tempo de amostragem de medição da corrente da lâmpada foi escolhido igual a $10 \mathrm{~ms}$, que corresponde ao período de acionamento em baixa frequiência. Esta escolha pode ser considerada aceitável para este tipo de aplicação. A função de transferência do controlador PI proposto é:

$$
R(s)=\frac{d(s)}{e(s)}=\frac{G_{a}}{s},
$$

em que $G_{a}$ é o ganho escalar e $e(s)$ a transformada do erro.

A Figura 6 mostra as características do sistema de malha fechada para $G_{a}=12,5$. A margem de fase é próxima de $90^{\circ}$, o que assegura um comportamento estável do sistema. O tempo de resposta é de aproximadamente $100 \mathrm{~ms}$. Este tempo de resposta pode ser considerado adequado porque a dinâmica do sistema é bastante lenta, da ordem de dezenas de segundos a minutos. Ou seja, um tempo de acomodação de $100 \mathrm{~ms}$ não interfere na luminosidade perceptível da lâmpada, nem em sua temperatura.

A discretização do regulador é representada na equação (9), enquanto a equação de diferença é descrita em (10) e a correspondente para $G_{a}=12,5$ é indicada em (11).

$$
\begin{gathered}
R(z)=\frac{d(z)}{e(z)}=\frac{A \cdot T_{m}}{z-1}=\frac{A \cdot T_{m} \cdot z^{-1}}{1-z^{-1}} . \\
D_{k}=D_{k-1}+A \cdot T_{m} \cdot \varepsilon_{k-1} . \\
D_{k}=D_{k-1}+\frac{1}{8} \varepsilon_{k-1} .
\end{gathered}
$$

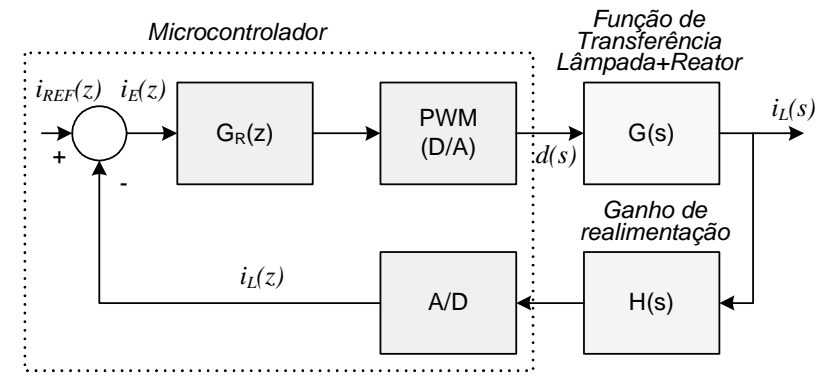

Figura 5: Diagrama de blocos do sistema de controle.

Em que $e(z)$ é a função de transferência digital do erro, $T_{m}$ é o tempo de amostragem e $\varepsilon_{k-1}$ o erro discretizado.

O controlador digital foi simulado usando a ferramenta $\mathrm{Si}$ mulink do Matlab, conforme ilustra a Figura 7(a). Por outro lado, a Figura 7(b) mostra os resultados da simulação, onde as respostas ao degrau e a uma perturbação podem ser verificadas. Para o primeiro caso, pode-se observar que a saída segue perfeitamente a referência com erro nulo, com um tempo de resposta de aproximadamente 100ms (similar a um controlador analógico). Quanto à perturbação programada em 200ms, pode-se observar que o controlador também compensa o erro em 100ms. Como já mencionado, se a lâmpada desligar durante esta fase, o microcontrolador esperará 5 minutos para reiniciar o processo e incrementar o contador G.

\subsection{Proteções}

Além das funções de controle básico descritas previamente, o microcontrolador deve oferecer algumas proteções cruciais para assegurar um comportamento confiável do reator, que são descritas a seguir.

1. Proteção Contra Falha de Partida, Lâmpada Danificada ou Ausência de Lâmpada: Se a lâmpada desligar durante algumas das fases de operação do reator, a tensão de saída começa a elevar-se, podendo alcançar níveis perigosos para os componentes do sistema. Conseqüentemente, duas proteções diferentes foram executadas durante as rotinas de medida de $V_{B U S}$ e de $i_{L}$. Se o microcontrolador detectar que a tensão de barramento superou $750 \mathrm{~V}$, ou se a corrente da lâmpada for mais baixa do que um valor mínimo, o sinal PWM é desligado, e o controle esperará 5 minutos antes de tentar um processo de partida novamente.

2. Proteções de Sobrecorrente: Os MOSFETs $M_{1}, M_{2}$ e $M_{3}$ devem ser protegidos contra sobrecorrente. Os interruptores de baixa freqüência, $M_{2}$ e $M_{3}$, já têm uma proteção inerente contra sobrecorrente devido ao mo- 


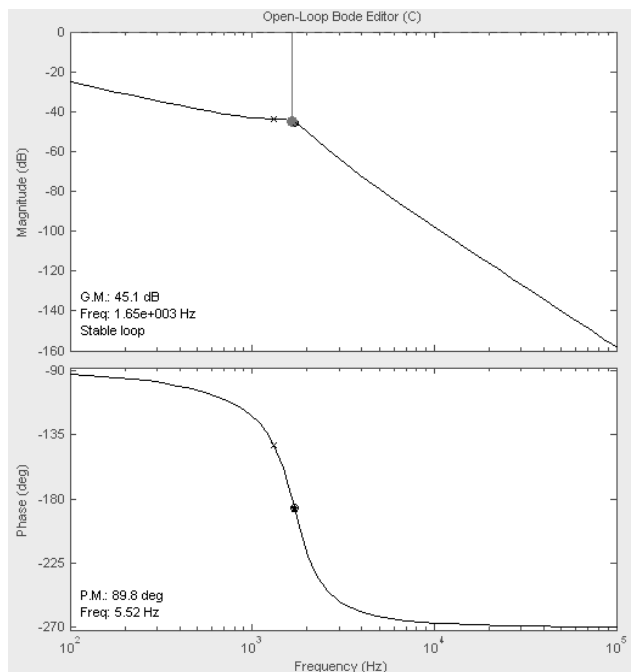

(a)

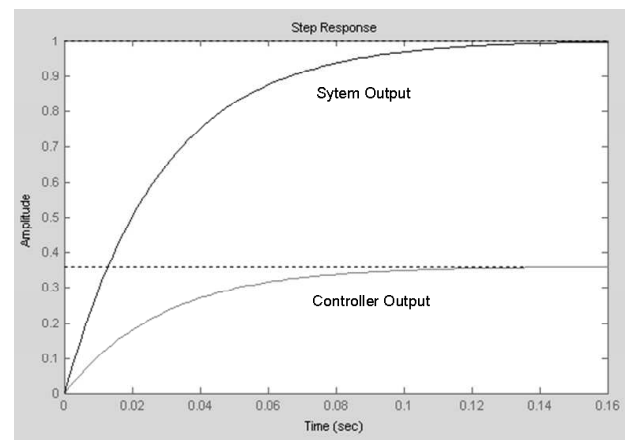

(b)

Figura 6: Característica do sistema de controle em malha fechada considerando $G_{a}=12,5$ : a) Diagramas de Bode e b) Resposta ao degrau.

nitoramento da corrente da lâmpada, $i_{L}$, uma vez que estes interruptores estão em série com a lâmpada.

Já a proteção de sobrecorrente de $M_{1}$ é executada por meio de um pedido da interrupção do microcontrolador. Se algumas destas proteções forem ativadas, o sinal de PWM será desligado, e o microcontrolador espera 5 minutos antes de tentar um processo de partida novo.

As variáveis de entrada e saída e alguns sinais de monitoramento e proteção podem ser vistos na Figura 4. $C_{A F}$ é o sinal do gerador PWM; $C_{B F}$ e seu nível lógico invertido são os sinais de acionamento em baixa freqüência; $i_{L}$ é o sinal de monitoramento da corrente da lâmpada; $V_{B U S}$ é a tensão de barramento, que corresponde a duas vezes a tensão da lâmpada; $C_{L}$ aciona um LED para indicar a necessidade de troca de lâmpada e $i_{L I M}$ é um sinal de interrupção em caso de sobrecarga em $M_{1}$.

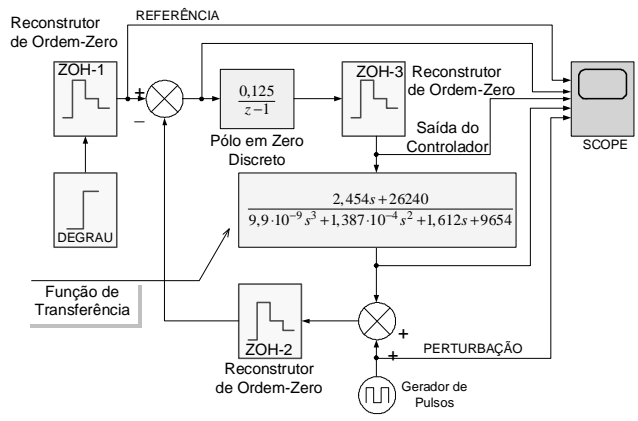

(a)
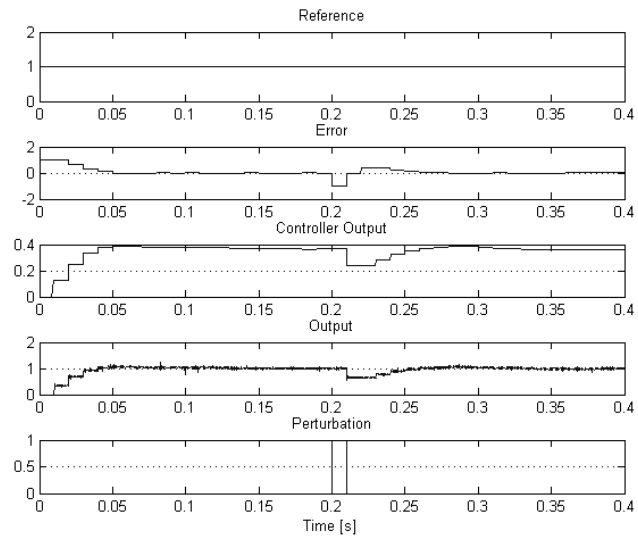

(b)

Figura 7: Simulação do regulador digital. (a) Circuito Simulado; (b) Resultados de simulação.

A Figura 8 mostra o fluxograma da estratégia de controle proposta, que resume as fases de operação do reator eletrônico. A Figura 9 mostra a operação teórica de partida sem falha de uma lâmpada, que pode ser descrita em quatro estágios diferentes, tomando-se como base as informações do fluxograma da Figura 8:

Estágio I) a lâmpada está desligada e então o microcontrolador aplica uma razão cíclica mínima, a fim de manter a tensão do barramento em torno de 700V. Conseqüentemente, o capacitor $C_{i g n}$ se carregará até a tensão de limiar do centelhador. Quando o centelhador conduz, um pulso de ignição é aplicado à lâmpada e a tensão do barramento cai rapidamente.

Estágio II) quando o microcontrolador detecta que a tensão do barramento cai abaixo de $400 \mathrm{~V}$ ele comanda um aumento na razão cíclica de $M_{1}$, fazendo $d=d_{W A R M}$, a fim manter a corrente da lâmpada e reduzir o tempo de ionização inicial. Esta fase continua até que o reator atinja o modo crítico de condução, quando a lâmpada apresentará sua tensão mínima, $V_{\text {Lmin }}$. 


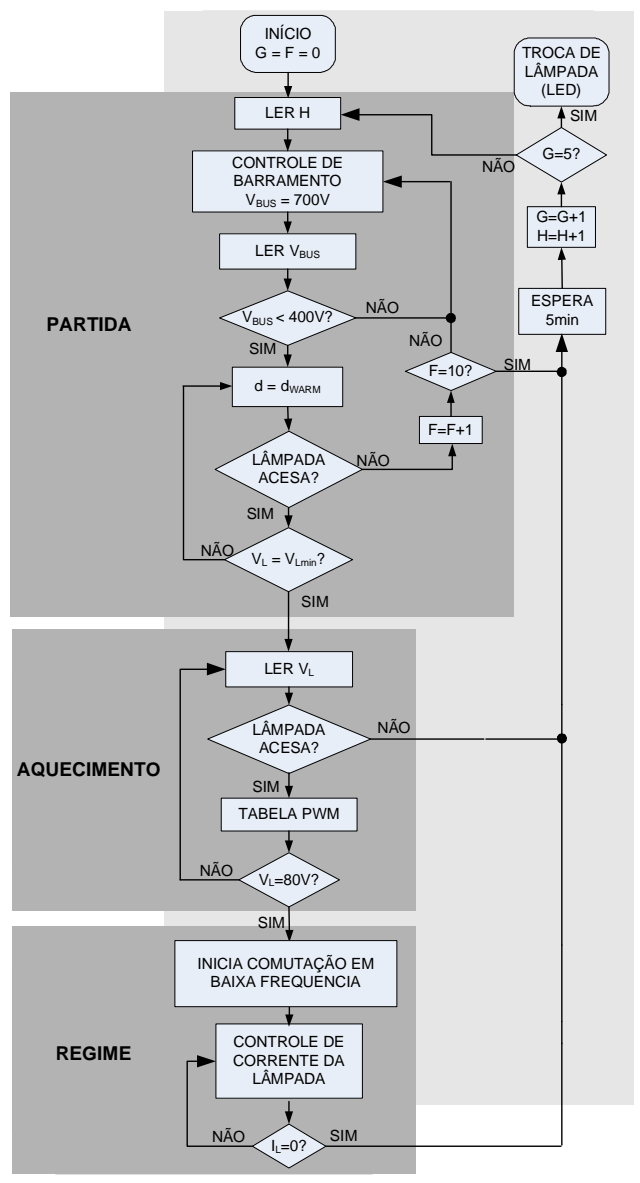

Figura 8: Fluxograma que resume a estratégia de controle proposta.

Estágio III) quando a tensão da lâmpada cai abaixo de um valor mínimo, o microcontrolador controla a razão cíclica usando uma tabela armazenada em sua memória interna que relaciona a largura de pulso com a tensão de barramento. Isto faz com que a lâmpada se aqueça com uma energia máxima, o que aumenta sua vida útil, garantindo a operação DCM do conversor flyback.

Estágio IV) quando a tensão da lâmpada alcança seu valor nominal (que varia de 80 a $100 \mathrm{~V}$ ), o microcontrolador dá início ao acionamento em baixa freqüência dos interruptores $M_{2}$ e $M_{3}$, e passa a controlar a corrente da lâmpada com base no controlador digital PI. Se o microcontrolador detectar, em alguns dos estágios, que a tensão da barramento alcança 750 V (por exemplo: se a lâmpada desligar), o sinal PWM é desativado. Neste caso, espera-se 5 minutos para reiniciar o processo de partida da lâmpada. Como já mencionado, este limite da tensão é selecionado porque os transistores $M_{2}$ e $M_{3}$ possuem uma tensão nominal de $800 \mathrm{~V}$.

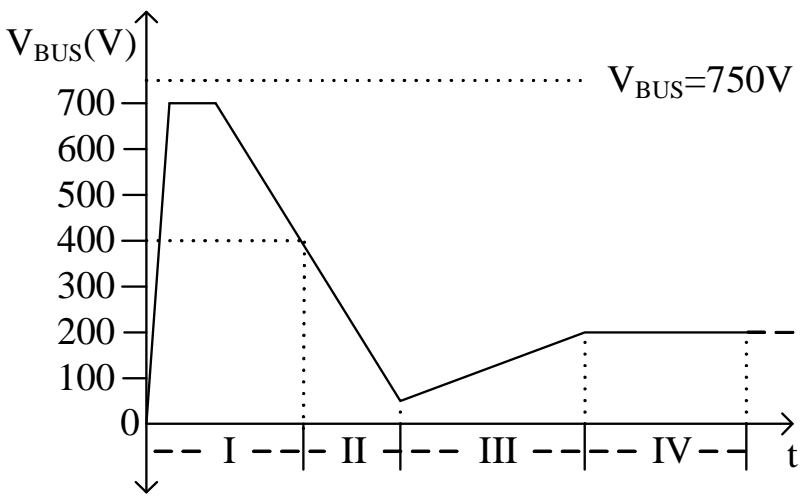

Figura 9: Etapas teóricas de partida da lâmpada.

\section{RESULTADOS EXPERIMENTAIS}

Esta seção apresenta os resultados experimentais do circuito proposto, a fim ilustrar a estratégia de controle executada pelo microcontrolador. A Figura 10 mostra os pulsos de tensão de ignição aplicados à lâmpada quando o centelhador conduz para as condições de: (a) sem lâmpada, e (b) com lâmpada. Como pode ser observado, o pulso na ausência de lâmpada alcança mais de $4 \mathrm{kV}$ e tem uma duração superior a $1 \mu \mathrm{s}$, o que atende às normas. Como pode ser visto na Figura 10(b), que descreve a condição com lâmpada, quando a tensão aplicada atinge o valor de ignição, a tensão na lâmpada reduz-se drasticamente, caracterizando assim um processo de partida bem-sucedido.

A Figura 11 mostra um detalhe do processo de partida. Quando o pulso da ignição é aplicado à lâmpada, a tensão de barramento diminui abaixo de $400 \mathrm{~V}$, e a razão cíclica é aumentada de aproximadamente 10 a $20 \%$. Este procedimento é muito importante para manter a lâmpada ligada e para assegurar um rápido aquecimento da lâmpada. O processo de aquecimento completo é mostrado na Figura 12, que destaca as alterações na razão cíclica (mediante consulta à tabela armazenada na memória do microcontrolador). Pode-se observar que, pela atuação do sistema de controle, a corrente da lâmpada não atinge valores excessivos durante a fase de aquecimento, apresentando um valor similar àquele observado no estado permanente. Este comportamento implica na conclusão da etapa de aquecimento em menos de 3 minutos, o que assegura uma vida longa à lâmpada, evitando uma degradação excessiva de seus elétrodos.

A Figura 13 mostra a tensão e corrente de linha, denotando o fator de potência elevado, que é de aproximadamente 0,96 . As componentes harmônicas cumprem as exigências da norma IEC61000-3-2. Tal conclusão está claramente fundamentada na Tabela II, que informa a amplitude das componentes harmônicas da corrente de linha, seu valor relativo à fundamental e os limites da norma. 


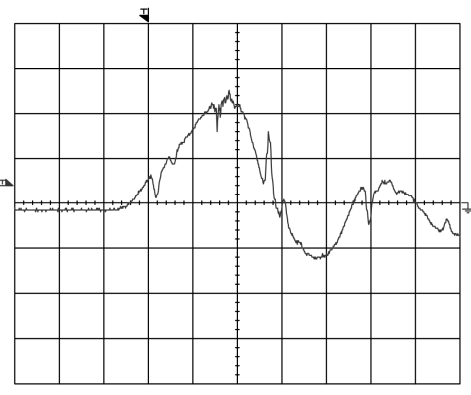

(a) Lâmpada ausente (2000V/div; 500ns/div).

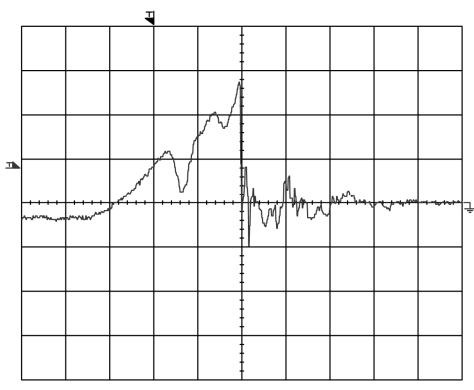

(b) Lâmpada presente (1000V/div; 200ns/div).

Figura 10: Pulsos de ignição gerados pelo reator proposto.

A Figura 14 mostra as formas de onda quadradas de baixa frequiência de tensão e corrente na lâmpada. Não se observou qualquer comportamento instável devido a ressonâncias acústicas. Apesar de se poder observar alguns picos discretos de tensão sobre a lâmpada (devido à indutância de magnetização do transformador flyback), percebe-se que eles não aparecem na corrente correspondente, o que garante um fator de crista muito baixo preservando-se, assim, a vida útil da unidade.

A Figura 15 mostra a resposta em malha-fechada da corrente da lâmpada para um ensaio de perturbação na tensão de entrada. Pode-se observar como a corrente da lâmpada é regulada em aproximadamente $80 \mathrm{~ms}$, que é o tempo de resposta previsto do regulador projetado.

A eficiência total medida do circuito é de aproximadamente 90\%. Contudo, uma avaliação mais detalhada sobre este ponto pode ser realizada admitindo-se uma variação de tensão de entrada de $+/-20 \%$ em torno da tensão nominal, de 230Vrms. No caso da tensão máxima de entrada, a eficiência observada foi de $91,2 \%$, ao passo que para a tensão mínima, mediu-se $88,4 \%$. Este desempenho só é alcançado em função da integração de estágios promovida pela topologia proposta, o que garante um nível reduzido de perdas de comutação e condução.

Tabela 2: Comportamento Harmônico da Corrente de Linha.

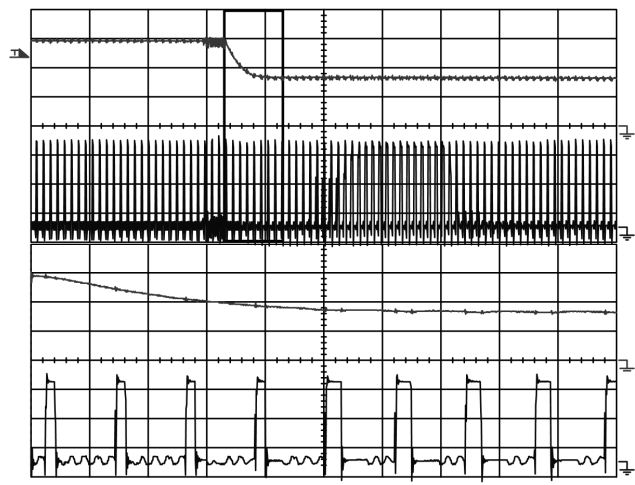

Figura 11: Em cima: VBUS e pulsos de alta freqüência (200V/div; 5V/div; and 100?s/div). Em baixo: zoom com $10 ? \mathrm{~s} / \mathrm{div}$.

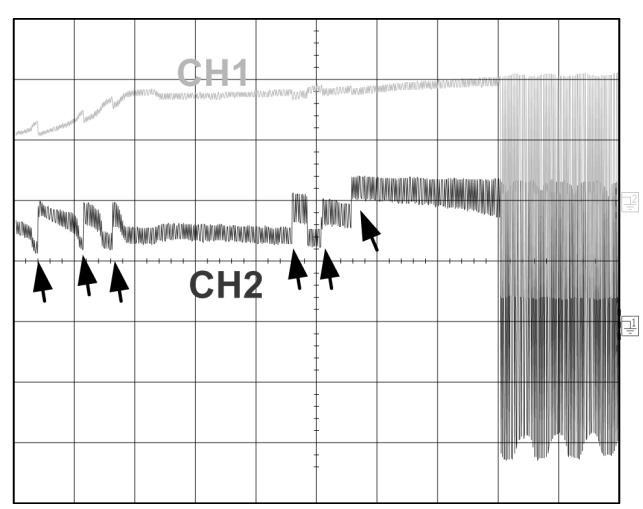

Figura 12: Processo de aquecimento completo: tensão na lâmpada (CH1) e corrente (CH2) (50V/div; 200mA/div; 20s/div).

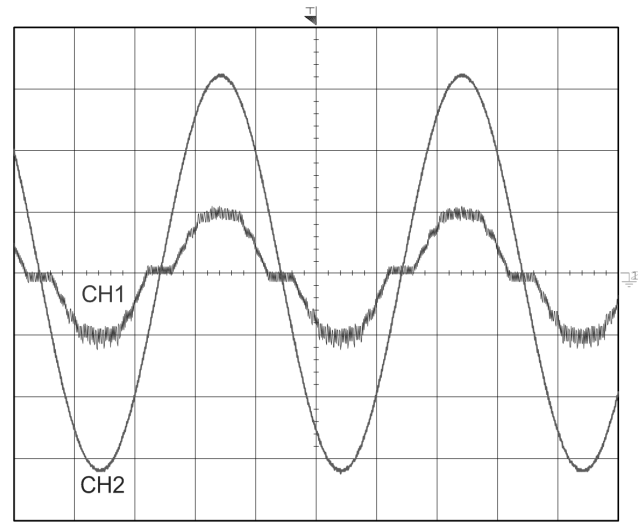

Figura 13: Tensão $(\mathrm{CH} 2)$ e corrente de linha $(\mathrm{CH} 1)$ (100V/div; 0,5A/div; 5ms/div). 


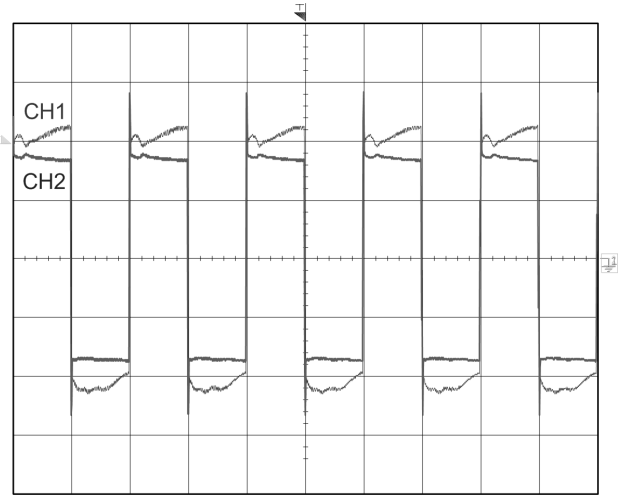

Figura 14: Tensão $(\mathrm{CH} 2)$ e corrente na lâmpada $(\mathrm{CH} 1)$ (50V/div; 0,2A/div; 5ms/div).

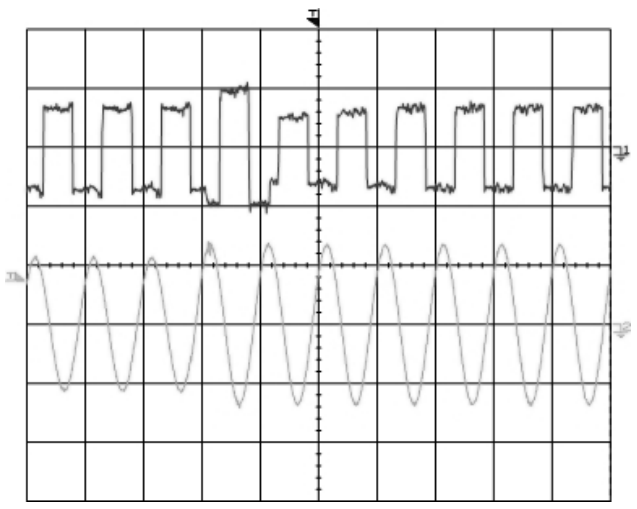

Figura 15: Resposta a perturbação na tensão de entrada: Em cima: corrente na lâmpada $(0,5 \mathrm{~A} / \mathrm{div}, 20 \mathrm{~ms} / \mathrm{div}), \mathrm{Em}$ baixo: tensão de linha (250V/div, 20ms/div).

\section{CONCLUSÕES}

Este artigo descreveu os detalhes de uma estratégia de controle digital, baseada em microcontrolador, aplicada a um reator eletrônico de elevado fator de potência. Tal reator foi projetado para acionar uma lâmpada de vapor metálico e emprega a técnica de integração de estágios. A topologia do conversor estático integra um estágio pré-regulador buck (que opera no modo descontínuo e corrige o fator de potência do reator) com um conversor flyback (que fornece potência à lâmpada em baixa frequiência). O microcontrolador garantiu flexibilidade na partida da lâmpada $\mathrm{MH}$, um adequado processo de aquecimento, condições de operação segura em estado permanente e respectivas proteções; que são tarefas relativamente complexas.

Um microcontrolador PIC 16F684 foi empregado para executar as tarefas de controle do sistema. A partida da lâmpada foi obtida por meio de um dispositivo centelhador (sparkgap) que gera os pulsos de ignição somente quando a lâm-
Tabela 2: Comportamento Harmônico da Corrente de Linha.

\begin{tabular}{|c|c|c|c|}
\hline $\begin{array}{c}\text { Ordem } \\
\text { Harmônica }\end{array}$ & $\begin{array}{c}\text { Valor } \\
\text { Eficaz } \\
(\mathbf{m A})\end{array}$ & $\begin{array}{c}\text { Valor Relativo } \\
\text { à Fundamental } \\
(\boldsymbol{\%})\end{array}$ & $\begin{array}{c}\text { Norma } \\
(\%)\end{array}$ \\
\hline 2 & 0 & 0 & 2 \\
\hline 3 & 25 & 14,12 & $\begin{array}{c}30 x 0,96 \\
=28,8\end{array}$ \\
\hline 5 & 15 & 8,47 & 10 \\
\hline 7 & 5 & 2,82 & 7 \\
\hline 9 & 3 & 1,69 & 5 \\
\hline 11 & 1 & 0,56 & 3 \\
\hline 13 & 4 & 2,26 & 3 \\
\hline 15 & 2 & 1,13 & 3 \\
\hline 17 & 1 & 0,56 & 3 \\
\hline 19 & 3 & 1,69 & 3 \\
\hline 21 & 1 & 0,56 & 3 \\
\hline 23 & 3 & 1,69 & 3 \\
\hline 25 & 1 & 0,56 & 3 \\
\hline 27 & 1 & 0,56 & 3 \\
\hline 29 & 0 & 0 & 3 \\
\hline 31 & 1 & 0,56 & 3 \\
\hline 33 & 0 & 0 & 3 \\
\hline 35 & 0 & 0 & 3 \\
\hline 37 & 1 & 0,56 & 3 \\
\hline 39 & 0 & 0 & 3 \\
\hline & & & \\
\hline
\end{tabular}

pada está desligada. Isto ocorre porque, quando o arco na lâmpada se estabelece, a condução do centelhador não é mais possível, devido à diminuição de $V_{B U S}$. A fim de controlar a lâmpada no estado permanente, um controlador digital PI foi executado por software, mantendo a corrente da lâmpada constante durante sua vida útil.

O reator proposto apresentou fator de potência elevado, condições factíveis de implementação, eficiência elevada e confiabilidade. Adicionalmente, o algoritmo de controle proposto pode ser empregado em outras topologias eletrônicas de reatores, bem como adaptado a outras lâmpadas de descarga de alta intensidade (HID).

\section{AGRADECIMENTOS}

Os autores desejam agradecer à CAPES e ao Ministério de Educação e Ciência da Espanha (Projeto DPI-2003-00308) que apoiaram a realização deste trabalho através da conces- 
são de bolsas de estudo a dois autores (Henrique A. C. Braga e Marco Dalla Costa, respectivamente).

\section{REFERÊNCIAS}

Alonso, J. M.; Calleja, A. J.; Ribas, J.; Corominas, E. L. \& Rico-Secades, M. (2004). Analysis and Design of a Novel Single-Stage High-Power-Factor Electronic Ballast Based on Integrated Buck Half-Bridge Resonant Inverter. IEEE Transactions on Power Electronics, vol. 19, no. 2, 550-559.

Alonso, J. M.; Villegas, P. J. ; Díaz, J.; Blanco, C. \& RicoSecades, M. (1997). A Microcontroller-Based Emergency Ballast for Fluorescent Lamps. IEEE Trans. on Industrial Electronics, vol. 44, no. 2, 207-217.

Alonso, J. M.; Dalla Costa, M. A.; Cardesín, J.; MartínRamos, J. A. \& García-García, J. (2007). Small-Signal Modeling of Discharge Lamps through Step Response and its Application to Low-frequency SquareWaveform Electronic Ballasts. IEEE Trans. on Power Electronics, vol. 22, no. 3, 744-752.

Bhat, A. K. \& Venkatraman, R. (2005). A Soft-Switched Full-Bridge Single-Stage AC-to-DC Converter With Low-Line-Current Harmonic Distortion. IEEE Transactions on Industrial Electronics, vol. 52, 1109-1116.

Buso, S., Mattavelli; P., Rossetto, L. \& Spiazzi, G. (1998). Simple Digital Control Improving Dynamic Performance of Power Factor Preregulators. IEEE Transactions on Power Electronics, vol. 13, no.5, 814-823.

Có, M. A.; Brumatti, M.; Simonetti, D. S. L. \& Vieira, J. L. F. (2003). Single Stage Electronic Ballast for HID Lamps. Proceedings of the IEEE Industry Applications Conference (IAS '03), vol. 1, 339-344.

Dalla Costa, M. A.; Alonso, J. M.; García, J. \& Cardesín, J. (2006). Analysis, Design and Experimentation of a Closed-Loop Metal Halide Lamp Electronic Ballast. Proceedings of the IEEE Industry Applications Conference (IAS'06), vol. 3, 1384-1390.

Dalla Costa, M. A.; Alonso, J. M.; García, J., Cardesín, J. \& Rico-Secades, M. (2005). A Novel Low-Cost Electronic Ballast to Supply Metal Halide Lamps. 40th IEEE IAS Annual Meeting (IAS'05), vol. 2, Kowloon, Hong Kong, 1198-1204.

Dalla Costa, M. A.; Alonso, J. M.; García, J., Cardesín, J. \& Rico-Secades, M. (2007). Acoustic Resonance Characterization of Low-Wattage Metal Halide Lamps under Low-Frequency Square-Waveform Operation. IEEE Transactions on Power Electronics, vol. 22 , no. 3, 735- 743 .
Dalla Costa, M. A.; Alonso, J. M.; Ribas, J., Cardesín, J. \& García, J. (2007). Acoustic-Resonance Characterization of Low-Wattage Metal-Halide Lamps. IEEE Transactions on Plasma Science, vol. 35, no. 1, 43-58.

de Groot, J. J. \& van Vliet, J. A. J. M. (1986). The HighPressure Sodium Lamp, Philips technical library. MacMillan Education, 1986.

Deng, E. \& Æuk, S. (1997). Negative Incremental Impedance and Stability of Fluorescent Lamps. IEEE Applied Power Electronics Conference (APEC '97), Vancouver, Canada: pp. 1050-1056.

Feng, Q.; Nelms, R. M. \& Hung, J. Y. (2006). Posicast-Based Digital Control of the Buck Converter. IEEE Transactions on Industrial Electronics, vol. 53, no. 3, 759-767.

Kutluay, K.; Çadirci, I.; Yafavi, A. \& Çadirci, Y. (2006). Dual 8-b Micro-Controllers - Digital Control of Universal Telecommunication Power Supplies. IEEE Industry Applications Magazine, vol. 12, no 1, 59-67.

Lee, K. C. \& Cho, B. H. (2003). Design and Analysis of Automotive High Intensity Discharge Lamp Ballast Using Micro Controller Unit. IEEE Trans. On Power Electronics, vol. 18 , no. 6, 1356-1364.

Li, H.; Shen, M. \& Qian, Z. (2004). A Novel Low Frequency Electronic Ballast for HID Lamps, Proceedings of the IEEE Industry Applications Conference (IAS '04), vol.: 1, 3-7, 321-324.

Marchesan, T. B.; Dalla Costa, M. A.; Alonso, J. M. \& do Prado, R. N. (2007). Analysis and Design of Two Flyback-Based Integrated Converters for the Implementation of LFSW Electronic Ballasts. Congresso Brasileiro de Eletrônica de Potência, COBEP 2007, Anais eletrônicos em CD-ROM, Blumenau-SC, 30 de setembro a 4 de outubro de 2007.

Marques, L. S., Sá Jr.; E. M. \& Perin, A. (2007). Topologic Study of Ballast Drives for a D2S-35W Lamp. Congresso Brasileiro de Eletrônica de Potência, COBEP 2007, Anais eletrônicos em CD-ROM, Blumenau-SC, 30 de setembro a 4 de outubro de 2007.

Shen, M.; Qian, Z. \& Peng, F. Z. (2003). Design of a TwoStage Low-Frequency Square-Wave Electronic Ballast for HID Lamps. IEEE Trans. on Industry Applications, vol. 39, no. 2, 424-430.

Wu, T. F.; Hung, J. C.; Tseng, S. Y. \& Chen, Y. M. (2005). A Single-Stage Fast Regulator with PFC based on an Asymmetrical Half-Bridge Topology. IEEE Transactions on Industrial Electronics, vol. 55, 139-150. 
Yan, W.; Hui, S. Y. R. \& Ho, Y. K. E. (2001). Stability Study and Control Methods for Small-Wattage HighIntensity-Discharge (HID) Lamps. IEEE Trans. on Industry Applications, vol. 37, no. 5, 1522-1530.

Yang, Y.; Qian, Z. \& Wu, X. (2005). A Novel SingleStage Low-Frequency Square-Wave Electronic Ballast for Low-Wattage HID Lamps. Proceedings of the IEEE Applied Power Electronics Conference (APEC '05), vol. 1, 1048-1052.

Zobaa, A. F. (2006). Maintaining a Good Power Factor and Saving Money for Industrial Loads. IEEE Transactions on Industrial Electronics, vol. 53, 710-711. 